\title{
Detecting Semantic Relations Between Nominals Using Support Vector Machines and Linguistic-Based Rules
}

\author{
Isabel Segura-Bedmar, Doaa Samy, Jose L. Martínez-Fernández, \\ and Paloma Martínez \\ Universidad Carlos III de Madrid, Computer Science Departament, \\ Avd. Universidad, 30, Leganes, 28911, Madrid, Spain \\ \{isegura, dsamy, jlmferna, pmf \}@inf.uc3m.es
}

\begin{abstract}
This paper describes the improvement of an automatic system for detecting semantic relations between nominals by the use of linguistically motivated knowledge combined with machine learning techniques. A previous version of the system using a Support Vector Machine classifier was evaluated in the $4^{\text {th }}$ International Workshop on Semantic Evaluations, SEMEVAL [5]. The performance of the system improved significantly by the application of the linguistic based rules.
\end{abstract}

Keywords: computational semantics, semantic relations, classification, Support Vector Machines, Sequential Minimal Optimization.

\section{Introduction}

The enormous growth of Internet in the last decade has produced a growing interest in the automatic processing of written text, especially from the semantic perspective. Ontology development to support Semantic Web has lead to the improvement of techniques capable of managing underlying semantic relations between concepts. Detecting semantic relations is a key issue in automating the ontology learning process. Techniques to automatically locate these concepts and the relations between them have been the object of many studies. Following this research line, [3] identifies four techniques to automatically extract semantic relations from texts: pattern-based techniques [1], association rules [3], conceptual clustering and ontology learning from schemata [2]. In many cases, combined approaches are used to detect concepts and relations among them [6].

Following a linguistic perspective, studies on classification of semantic relations can be grouped in two major classes. The first focuses on "complex nominals", while the second studies the relation between nouns in more complex and longer constructions either at the phrase, clause or sentence level. The majority of approaches lie in the first group.

At the linguistic level, semantic relationships represent a number of challenges. They are highly ambiguous and context dependent, where information on the syntactic or the

This work has been partially supported by the Regional Government of Madrid under the Research Network MAVIR (S-0505/TIC-0267). 
lexical level are usually not enough to discover the underlying relations. These relations are not syntactically, but semantically governed.

\section{The System}

The system described in the present paper is a hybrid supervised system integrating different resources: machine learning algorithms (support vector machines) and language resources (tokenizers, POS taggers, WordNet as a lexical-semantic resource and linguistic-based rules).

Our scope only considers nominals where constituents of the relation are formally tagged as nouns. The nominals can occur either on the phrase, clause or sentence level. This fact constitutes the major challenge in this task since most of the previous studies limited their approaches to certain types of nominals, namely noun compounds, nominalizations and nouns on the phrase level.

\subsection{The Data}

The $4^{\text {th }}$ International Workshops on Semantic Evaluation, SEMEVAL $2007^{1}$, created a benchmark dataset and evaluation task, Classification of Semantic Relations between Nominals, enabling researches to compare their algorithms.

In this task, a nominal is a noun, excluding named entities, or base noun phrase,. A base noun phrase is a noun and its premodifiers (nouns, adjetives, determiners).

The dataset included seven semantic relatons: Cause-Effect, Instrument-User, Product-Producer, Origin-Entity, Purpose-Tool, Part-Whole, Content-Container.

For each relation, 140 training sentences, 70 testing sentences and a precise definition of the relation were provided by the organizers. Each sentence was manually annotated including the following information: the nominal boundaries (e.g., Put $<e 1>$ tea $</ e 1>$ in $a<e 2>$ heat-resistant jug $</ e 2>$ ), the used query to search the sentence in the web (e.g., "* in $a *$ ") and the Wordnet sense key for each nominal (e.g., WordNet(e1) = "tea\%1:13:00::", WordNet(e2) = "jug\%1:06:00::").

\subsection{Support Vector Machines}

The set of features used for the classification of semantic relations includes information from different levels: word tokens, POS tags, verb lemmas, semantic information from WordNet, etc.

Information regarding word tokens, POS tags and lemmas are automatically extracted using the using infrastructure for developing software components for Language Engineering GATE ${ }^{2}$. Lexical features are related to both the nominals and the contextual information where certain features keep track of the words occurring before, after and between the nominals. Contextual features include the two words before the first nominal, the two words after the second nominal and the word list inbetween. Another feature, called numinter, indicating the number of words between nominals is also considered.

\footnotetext{
${ }^{1}$ http://nlp.cs.swarthmore.edu/semeval/

${ }^{2}$ http://gate.ac.uk/
} 
Features regarding the POS level focus on the contextual information. The system extracts the POS tags of the words occurring before and after the nominals. In addition, another feature includes the path from the first nominal to the second nominal. This path feature is built by the concatenation of the POS tags of the words occurring between both nominals. In case the list of words occurring in between the two nominals include a verb or a preposition, more morphological features are considered such as the lemma and the voice in case of a verb and the lemma and type in case of a preposition.

At the semantic level, we used features obtained from WordNet and already provided in the training and testing data. This information includes the synset number and the lexical file number for each nominal. For the Part-Whole relation, we use an additional feature indicating the meronymy relation between the two nominals.

The set of WordNet lexical files can help to determine if the nominals satisfy the restrictions for each relation. For example, in the relation Theme-Tool, the theme should be an object, an event, a state of being, an agent, or a substance. Else, it is possible to affirm that the relation is false.

An additional feature, called WordNet vector was designed. It is a 13 dimension vector where each coordinate represents one of the 13 nodes in the third level of depth in WordNet. We use a binary representation, i.e. if the synset is ancestor of the nominal it is assigned the value 1 , else it is assigned the value 0 . Our initial hypothesis considers that this representation for the nominals could perform well on unseen data. Finally, features related to the query provided for each sentence are also taken into consideration.

Support Vector Machines (SVM) model was selected for its good performance in many classification tasks such as Semantic Role Labeling and Document Categorization. The data is represented as an $n$-dimensional vector, and the final goal is finding the maximal separating hyperplane of dimension $n-1$ between the two classes (true and false). There are several algorithms for quickly finding the parameters of the hyperplane. We decided to use Sequential Minimal Optimization (SMO) [4]. SMO breaks the large quadratic programming (QP) optimization problem needed to be resolved in SVM into a series of smallest possible QP problems. These small QP problems are analytically solved, avoiding, in this way, a time-consuming numerical QP optimization as an inner loop. We used Weka [7] an implementation of the SMO.

Both run time and accuracy depend critically on the values given to two parameters: the upper bound on the coefficient's values in the equation for the hyperplane $(-\mathrm{C})$, and the degree of the polynomials in the non-linear mapping (-E) [7]. The best settings for a particular dataset can be found only by experimentation. We did not experiment with the polynomial kernel. The default value is set to 1 . However, we made numerous experiments to find the best value for the parameter $\mathrm{C}(\mathrm{C}=1, \mathrm{C}=10$, $\mathrm{C}=100, \mathrm{C}=1000, \mathrm{C}=10000$ ), but the results were not remarkably affected. Probably, this is due to the small size of the training set.

\subsection{Rules}

Parallel to the work on training the machine learning model, a set of linguistic and knowledge based constraint rules were manually developed. Our main concern, in this respect, is not the coverage of the rules, but the improvement achieved by applying 
them. Thus, the constraint rules do not pretend to achieve a total coverage of the whole dataset, but to improve the results of the learning algorithm in cases where it is obvious to decide if the relation is true or false according to simple semantic and knowledge constraints.

For each semantic relation, a number of rules were developed based on the definition given by the SEMEVAL-task 4 and the analysis of the training dataset. In this analysis, we considered simple heuristics and linguistic features on the different levels (POS, lexical, syntactic and semantic). We counted on three basic sources of information, considered as basic criteria:

- WordNet Information concerning synsets and lexical file numbers of each nominal in the WordNet structure.

- POS information mainly lemmas, grammatical categories and lemmas, provided from the basic processing module.

- Lexical information regarding the word tokens occurring in the sentences are also provided in the basic processing module.

It is important to highlight the fact that we did not use a syntactic analyzer or a chunker. Thus, the syntactic information, included in the rules, consists basically of simple sequences of POS tags. In this respect, we believe that integrating information concerning chunks or basic syntactic analysis in future versions would be highly valuable as we will discuss later.

Taking into account the above mentioned criteria, we can state that our rules combine semantic information with lexical and morpho-syntactic information. These perspectives are not mutually exclusive. On the contrary, they do overlap in many ways, revealing the complexity of natural language since it adopts a variety of mechanisms to reflect the relations between concepts. These mechanisms are manifested in different linguistic levels varying from the most abstract conceptual to the more precise lexical or morphological (nouns, verbs and prepositions) levels.

Rules developed can be divided into four basic classes: 1) semantic rules dealing with the most abstract level. These rules depend mainly on the lexical file numbers. 2) Lexico-semantic rules where special lemmas and tokens of the contextual information are taken into account. 3) morphosyntactic rules where special patterns consisting of sequences of POS are considered. 4) Complex rules combining two or more of the above mentioned criteria or addressing special grammatical phenomena such as the passive voice. In the following paragraphs, we will give examples of these rules among the different semantic relationships.

For the CAUSE-EFFECT relation, a set of four rules was developed. For example, the rule 1 belong to the first class of simple semantic rules. It states the following:

Rule Cause-Effect 1: IF Cause IS (TIME OR LOCATION OR PERSON OR ANIMAL OR GROUP OR COMMUNICATIVE ACT) THEN R(Cause-Effect)=False However, rule 3 and rule 4 are complex rules reflecting certain patterns where two sources of information are combined: information about tokens, lemmas and synsets of the nominals in concern and information concerning the words occurring in between.

Rule Cause-Effect 3: IF (CAUSE_TOKEN=EFFECT_TOKEN) AND (TOKEN_BETWEEN_NOMINALS= "after") THEN R (Cause-Effect)=False For example, "frustration after frustration" this pattern does not imply a cause-effect relation. However, it indicates a frequency. 
In many cases, the CAUSE-EFFECT relation might be expressed through the use of the preposition from indicating an EFFECT from a CAUSE. However, according to the case indicated by rule 4 , the use of the preposition is governed and implied by the noun and, thus, it looses its semantic connotation of CAUSE-EFFECT.

Rule Cause-Effect 4: If (CAUSE_TOKEN=="protection" (synset= 03969138 OR synset $=00805831$ ) OR (CAUSE_TOKEN=="abstinence" $($ synset $=01054457 \quad$ OR $\quad$ synset $=\quad$ 04827378) $) \quad$ AND (TOKEN_BETWEEN_NOMINALS $=$ "from") THEN R(Cause-Effect) $=$ False Other types of complex rules deal with grammatical phenomena such as the passive voice. The role of passiveness is crucial in indicating agency features since passiveness implies patience and denies agency. This linguistic fact is represented in a special rule for the INSTRUMENT-AGENCY relation indicating that using the passive voice negates the agency and, thus, the relation is false.

Rule Instrument-Agency 1: If ((NOMINAL_AGENCY + Verb Participle+ "with"+ NOMINAL_INSTRUMENT) OR (NOMINAL_AGENCY + Verb(be) + Verb_Participle+ "with" +NOMINAL_INSTRUMENT)) Then R(InstrumentAgency) $=$ False

For the PRODUCT-PRODUCER relation, other features were considered in developing the set of rules. The analysis of the training dataset revealed that this relationship is frequently represented in English language through the use of transitive verbs indicating actions like: \{make, produce, manufacture \}, \{develop, compose, prepare $\}$ or \{create, procreate $\}$. A complex rule is developed (combining lexical, semantical and syntactic through a sequence of POS tags) using morpho-syntactic and lexical information together with semantic information regarding the synsets of these verbs and simple semantic constraints indicating that PRODUCT cannot be TIME, STATE or ACTIVITY.

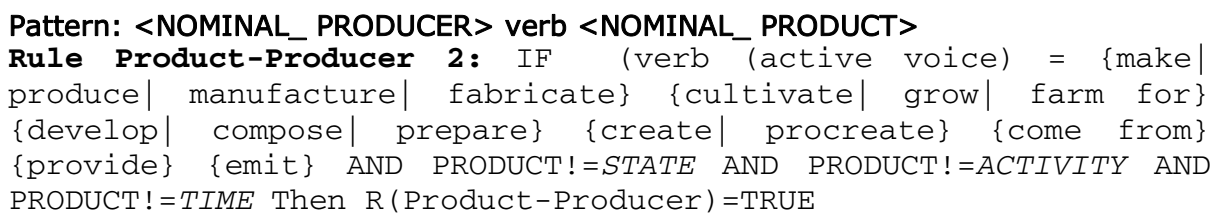

The order of executing the rules is essential; it goes from the most specific to the most generic in order to avoid false generalizations. If no lexico-syntactic patterns are available, we apply the general semantic-based rules For example; the above rule in the PRODUCT-PRODUCER relation implies another generic rule indicating the following:

Rule Product-Producer 1: IF PRODUCT IS (STATE OR ACTIVITY OR TIME) THEN R (Product-Producer) =FALSE

As we previously mentioned, the semantics of prepositions are, in many cases, responsible of determining the relation between the nominals. However, this could lead to ambiguous cases where the use of the preposition is governed by the preceding noun. For example, the preposition from is frequently used to indicate ORIGINENTITY relation where an ENTITY comes from an ORIGIN. Nevertheless, in cases where the noun preceding the preposition demands the use of from and the semantic content of the noun doesn't imply an ORIGIN-ENTITY relation, the relation is said to be false. This fact is expressed in a simple lexical rule: 
Pattern: VERB <NOMINAL_ENTITY> + PREP= "FROM" + <NOMINAL_ORIGIN> Rule Origin-Entity 1: IF verb $=$ \{separate $\mid$ prohibit $\mid$ protect save| avoid| shield| isolate Then R(Origin-Entity)=FALSE

Following these guidelines, a number of rules were developed according to the semantics implied in each relation. The coverage of these rules varied from one relation to another and the improvements obtained over the scores of the machine learning algorithm also varied as we will discuss in the next section.

\section{Experimental Results and Conclusions}

Introducing the rules enhanced overall scores in all relations except CONTENTCONTAINER as shown in Table1. Best results were obtained in the PRODUCTPRODUCER relation where the improvement reached almost $15 \%$ raising the overall accuracy from $60.8 \%$ to $75 \%$. This is due to the relatively high coverage of the rules $(48 \%)$ and their high precision. In CAUSE-EFFECT, rules improved the results approximately $4.5 \%$ raising the overall accuracy from $56.2 \%$ to $60.75 \%$. Again, the rules proved a high precision. For the INSTRUMENT-AGENCY, improvement was trivial (only 1.3\%) and, thus, we believe that more rules should be implemented. Results for ORIGIN-ENTITY relation was improved by a percentage of $5 \%$ reaching an overall accuracy of $70.5 \%$ in comparison with $65.4 \%$ achieved by the machine learning algorithm alone. For the THEME-TOOL relation, enhancement obtained is almost $6 \%$ improving the total accuracy from $54.2 \%$ to $60 \%$. It is also important to point out that the precision of the rules in this case was optimum $100 \%$ over the rule.

Finally in the PART-WHOLE relation, rules covered $36 \%$ of the test data. The improvement obtained reaches $6 \%$ enhancing the total accuracy to $76.38 \%$ after $70.8 \%$ obtained by the machine learning module (for this relation, our system was the first in the SEMEVAL task\#4).

The graph 1 shows the comparison between results obtained by the machinelearning module and those obtained after combining the rules.

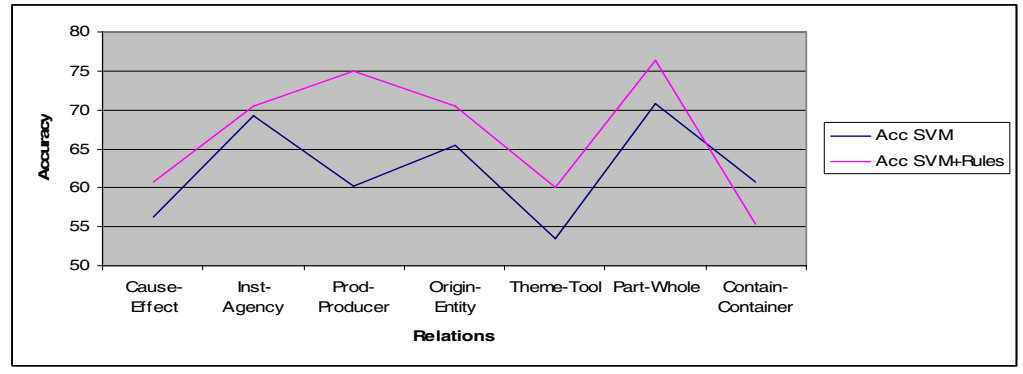

Graph 1. Compared Results between SVM and Hybrid Approach

Results obtained demonstrate that applying a hybrid approach on a supervised system achieves good results in a semantic classification task between nominals. Improvement obtained varies from $1.2 \%$ till $15 \%$ over the total accuracy scores obtained by the support vector machine algorithm, except for the relation CONTENTCONTAINER. 
Table 1. Number of Rules, Coverage and Results

\begin{tabular}{|c|c|c|c|c|c|}
\hline Relations & $\begin{array}{c}\text { Rules } \\
\text { (num) }\end{array}$ & $\begin{array}{c}\text { Coverage } \\
\text { Train }\end{array}$ & $\begin{array}{c}\text { Coverage } \\
\text { Test }\end{array}$ & $\begin{array}{c}\text { Acc. } \\
\text { SVM }\end{array}$ & $\begin{array}{c}\text { Acc. } \\
\text { SVM+Rules }\end{array}$ \\
\hline Cause-Effect & 4 & $25 \%$ & $27 \%$ & $56,2 \%$ & $60,75 \%$ \\
\hline Inst-Agency & 3 & $8 \%$ & $8 \%$ & $69,2 \%$ & $70,5 \%$ \\
\hline Prod-Producer & 7 & $50 \%$ & $48 \%$ & $60,2 \%$ & $75 \%$ \\
\hline Origin-Entity & 15 & $36 \%$ & $38 \%$ & $65,4 \%$ & $70,5 \%$ \\
\hline Theme-Tool & 6 & $38 \%$ & $25 \%$ & $53,5 \%$ & $60 \%$ \\
\hline Part-Whole & 45 & $62 \%$ & $36 \%$ & $70,8 \%$ & $76,38 \%$ \\
\hline C-Container & 15 & $16 \%$ & $18 \%$ & $60.8 \%$ & $55.40 \%$ \\
\hline
\end{tabular}

We want to introduce further training and enhancement. Improving results across the whole dataset requires wider use of semantic information such as hypernym for each synset or information regarding the entity features. Also, introducing more syntactic information through syntactic chunking could affect significantly the results. In the rule-based module, we consider increasing the set of rules for a broader coverage.

Semantic classification proposed by this system can be integrated in a Question Answering system or ontology building. For QA systems, to answer complex questions, a deeper understanding of the question is necessary. For example, to answer questions such as "list of all causes from asthma" (cause-effect), "list of all country producer of olive oil" (product-producer) or "what are the chemical components of aspirin? (Part-Whole). Detecting these types of semantic relations could help to find the exact answer.

\section{References}

1. Kietz, J.U., Maedche, A., Volz, R.: A method for semi-automatic ontology acquisiton from a corporare Intranet. In: Dieng, R., Corby, O. (eds.) EKAW 2000. LNCS (LNAI), vol. 1937, pp. 1-4. Springer, Heidelberg (2000)

2. Lee, D., CHU, W.W.: Constraints-Preserving Transformation from XML Document Type Definition to Relational Schema. In: Laender, A.H.F., Liddle, S.W., Storey, V.C. (eds.) ER 2000. LNCS, vol. 1920, Springer, Heidelberg (2000)

3. Maedche, A., Staab, S.: Measuring similarity between ontologies. In: Gómez-Pérez, A., Benjamins, V.R. (eds.) EKAW 2002. LNCS (LNAI), vol. 2473, Springer, Heidelberg (2002)

4. Platt, J. Sequential Minimal Optimization: A Fast Algorithm for Training Support Vector MachinesMicrosoft Research Technical Report MSR-TR-98-14 (1998)

5. Segura Bedmar, I., Samy, D., Martinez-Fernández, J.L.: UC3M: Classification of Semantic Relations between Nominals using Sequential Minimal Optimization. In: Proc. of SEMEVAL, ACL O7. Prague (2007)

6. Specia, L., Motta, E.: A hybrid approach for extracting semantic relations from texts. In: 2nd Workshop on Ontology Learning and Population (2006)

7. Witten, H., Frank, E.: Data Mining: Practical machine learning tools and techniques. Morgan Kaufmann, San Francisco (2005) 